\title{
An example related to Whitney extension with almost minimal $\mathrm{C}^{\mathrm{m}}$ norm
}

\section{Charles Fefferman and Bo'az Klartag}

\begin{abstract}
We present a counter-example to a certain conjecture that is related to Whitney's extension problems.
\end{abstract}

\section{Introduction}

Suppose that $F: \mathbb{R}^{n} \rightarrow \mathbb{R}$ is a $C^{m}$-function, that is, a function all of whose derivatives up to order $m$ exist and are continuous. The $\mathrm{C}^{\mathrm{m}}$ norm of $\mathrm{F}$ is frequently defined as

$$
\|F\|_{C^{m}}=\sup _{x \in \mathbb{R}^{n}} \max _{0 \leq|\alpha| \leq m}\left|\partial^{\alpha} F(x)\right|
$$

When the function $F$ is rotated, its $\mathrm{C}^{\mathrm{m}}$ norm might slightly change. Nevertheless, for any isometry $\mathrm{U}: \mathbb{R}^{n} \rightarrow \mathbb{R}^{n}$ we always have that $\|\mathrm{F} \circ \mathrm{U}\|_{\mathrm{C}^{m}} \leq$ $\mathrm{C}\|\mathrm{F}\|_{\mathrm{C}^{\mathrm{m}}}$, where $\mathrm{C}>0$ is a constant depending solely on $\mathrm{m}$ and $\mathrm{n}$. Thus, the $\mathrm{C}^{\mathrm{m}}$ norm, as defined above, is rotationally-invariant up to a constant. This is an example for a common characteristic of the use of the $\mathrm{C}^{\mathrm{m}}$ norm: In many cases, one is interested not in the exact quantity $\|F\|_{C^{m}}$, but in its "order of magnitude".

A result regarding the nature of the $\mathrm{C}^{\mathrm{m}}$ norm, in the "up to order of magnitude" scale, was recently obtained by the first named author [11]. It will be formulated next. See also Brudnyi-Shvartsman [7], whose earlier results and conjectures overlap with those of $[11]$. We write $\#(A)$ for the cardinality of the set $A$.

2000 Mathematics Subject Classification: 65D05, $65 \mathrm{D} 17$.

Keywords: Whitney extension, optimal norm, finiteness principle, counterexample. 
Theorem 1.1 Suppose $\mathrm{m}$ and $\mathrm{n}$ are positive integers. Let $\mathrm{E} \subset \mathbb{R}^{\mathrm{n}}$ be a finite set, $\mathrm{f}: \mathrm{E} \rightarrow \mathbb{R}$ and $\mathrm{M}>0$. Assume that for any subset $\mathrm{S} \subseteq \mathrm{E}$ with $\#(\mathrm{~S}) \leq \mathrm{k}^{\#}$ there exists a $\mathrm{C}^{\mathrm{m}}$-function $\mathrm{F}_{\mathrm{S}}: \mathbb{R}^{\mathrm{n}} \rightarrow \mathbb{R}$ such that

$$
\forall x \in S, F_{S}(x)=f(x) \quad \text { and } \quad\left\|F_{S}\right\|_{C^{m}} \leq M .
$$

Then, there exists a $\mathrm{C}^{\mathrm{m}}$-function $\mathrm{F}: \mathbb{R}^{\mathrm{n}} \rightarrow \mathbb{R}$ such that

$$
\forall x \in E, F(x)=f(x) \quad \text { and } \quad\|F\|_{\mathrm{C}^{m}} \leq \mathrm{CM} .
$$

Here, $\mathrm{C}, \mathrm{k}^{\#}>0$ are constants depending only on $\mathrm{m}$ and $\mathrm{n}$.

Theorem 1.1 pertains to Whitney's problem, going back to Whitney [27, 28, 29], with contributions by Glaeser [22], Brudnyi-Shvartsman [5],.., [9], [23, 24, 25], Zobin [30, 31], Bierstone-Milman-Pawłucki [1, 2], Fefferman $[10], \ldots,[19]$, A. and Y. Brudnyi [3] and Fefferman-Klartag [20, 21].

Motivated by the practical problem of multi-variate interpolation, an attempt to study the $\mathrm{C}^{\mathrm{m}}$ norm on a more accurate level was initiated by the first named author in $[18,19]^{1}$. Among the positive results obtained in these works, is an analog of the classical Whitney theorem for jets (see [27] or [26, Section VI]), with an accurate control of the $\mathrm{C}^{\mathrm{m}}$ norm. The investigations in $[18,19]$ take into account various possible definitions of $\mathrm{C}^{\mathrm{m}}$ norms, in addition to the definition (1.1). For instance, the results in $[18,19]$ are also applicable for the $\mathrm{C}^{\mathrm{m}}$ norm

$$
\|F\|_{C^{m}}=\sup _{x \in \mathbb{R}^{n}} \max _{k=0, \ldots, m}\left(\sum_{i_{1}, \ldots, i_{k}=1}^{n}\left|\frac{\partial^{k} F(x)}{\partial x_{i_{1}} \ldots \partial x_{i_{k}}}\right|^{2}\right)^{1 / 2} .
$$

The latter definition of a $\mathrm{C}^{\mathrm{m}}$ norm is exactly rotationally-invariant, and hence, perhaps, is slightly more natural than the definition (1.1). Of course, the definitions (1.1) and (1.2) are equivalent up to a constant depending only on $m$ and $n$. The finer analysis in $[18,19]$ has led to the formulation of the following optimistic conjecture.

Conjecture 1.2 Fix $\mathrm{m}, \mathrm{n}$ and a plausible definition of the $\mathrm{C}^{\mathrm{m}}$ norm (say, (1.1) or (1.2) above). Let $\mathrm{E} \subset \mathbb{R}^{n}$ be a finite set, $\mathrm{f}: \mathrm{E} \rightarrow \mathbb{R}$ and $\mathrm{M}, \varepsilon>0$.

Assume that for any subset $\mathrm{S} \subseteq \mathrm{E}$ with $\#(\mathrm{~S}) \leq \mathrm{k}^{\#}(\varepsilon)$ there exists a $\mathrm{C}^{\mathrm{m}}$-function $\mathrm{F}_{\mathrm{S}}: \mathbb{R}^{\mathrm{n}} \rightarrow \mathbb{R}$ such that

$$
\forall x \in S, F_{S}(x)=f(x) \quad \text { and } \quad\left\|F_{S}\right\|_{C^{m}} \leq M .
$$

Then, there exists a $\mathrm{C}^{\mathrm{m}}$-function $\mathrm{F}: \mathbb{R}^{\mathrm{n}} \rightarrow \mathbb{R}$ such that

$$
\forall x \in E, F(x)=f(x) \quad \text { and } \quad\|F\|_{C^{m}} \leq(1+\varepsilon) M .
$$

Here, $\mathrm{k}^{\#}(\varepsilon)>0$ is a constant depending only on $\mathrm{m}, \mathrm{n}, \varepsilon$ and the choice of the $\mathrm{C}^{\mathrm{m}}$ norm.

\footnotetext{
${ }^{1}$ However, we make no claims of practical significance for algorithms from [18, 19].
} 
Conjecture 1.2 admits a positive answer in the case $m=1$, for a large variety of $\mathrm{C}^{1}$ norms, as is explained in [18]. However, in this note we demonstrate that Conjecture 1.2 is false already in the first non-trivial case $\mathrm{m}=\mathrm{n}=2$, for most reasonable choices of a $\mathrm{C}^{2}$ norm. Thus, one is forced to look for more creative ways in order to estimate the $\mathrm{C}^{\mathrm{m}}$ norm of the best extension function, to an arbitrary precision.

The counter-example we present for Conjecture 1.2 takes a very simple geometric form. A schematic drawing of our set $E$ is shown in Figure 1.

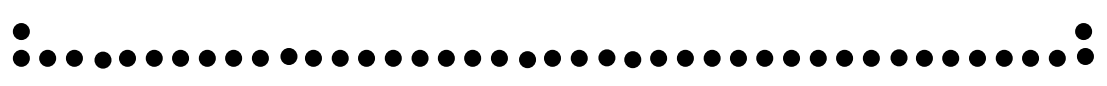

Figure 1

For the clarity of the exposition, in most of this article we will analyze our counter-example for a specific choice of the $C^{2}$ norm, that corresponds to the definition (1.2). In Section 6 we will comment on the adaptation of the argument to other $\mathrm{C}^{2}$ norms. Thus, from now on and until Section 6 , by $\|F\|_{C^{2}}$ we shall mean the following: For an open set $U \subseteq \mathbb{R}^{2}$ and a function $\mathrm{F}: \mathrm{U} \rightarrow \mathbb{R}$

$$
\|F\|_{C^{2}(u)}=\sup _{x \in U} \max _{k=0, \ldots, 2}\left(\sum_{i_{1}, \ldots, i_{k}=1}^{2}\left|\frac{\partial^{k} F(x)}{\partial x_{i_{1}} \ldots \partial x_{i_{k}}}\right|^{2}\right)^{1 / 2} .
$$

We abbreviate $\|\mathrm{F}\|_{\mathrm{C}^{2}}=\|\mathrm{F}\|_{\mathrm{C}^{2}\left(\mathbb{R}^{2}\right)}$. Thus, $\|\mathrm{F}\|_{\mathrm{C}^{2}}$ is invariant under translations, rotations and reflections in $\mathbb{R}^{2}$.

Proposition 1.3 For any positive integer $\mathrm{k}^{\#}$, there exists a finite set $\mathrm{E} \subset \mathbb{R}^{2}$ and a function $\mathrm{f}: \mathrm{E} \rightarrow \mathbb{R}$ with the following properties

1. For any $\mathrm{C}^{2}$-function $\mathrm{F}: \mathbb{R}^{2} \rightarrow \mathbb{R}$ with $\left.\mathrm{F}\right|_{\mathrm{E}}=\mathrm{f}$ we have that

$$
\|\mathrm{F}\|_{\mathrm{C}^{2}}>1+\mathrm{c}_{0} \text {. }
$$

2. For any subset $\mathrm{S} \subseteq \mathrm{E}$ with $\#(\mathrm{~S}) \leq \mathrm{k}^{\#}$, there exists a $\mathrm{C}^{2}$-function $\mathrm{F}: \mathbb{R}^{2} \rightarrow \mathbb{R}$ with

$$
\left.\mathrm{F}\right|_{S}=\mathrm{f} \text { and } \quad\|\mathrm{F}\|_{\mathrm{C}^{2}} \leq 1 .
$$

Here, $\mathrm{c}_{0}>0$ is a universal constant.

The proof of Proposition 1.3 is discussed in Sections 2-5. Throughout the proof, the letters $C, C^{\prime}, c, \tilde{c}$, etc. stand for various positive universal constants, whose value may change from one line to the next. We write Meas for Lebesgue measure on the real line. 


\section{Preliminaries}

The construction of our counter-example involves a certain universal constant from the classical Whitney extension theorem. The following lemma is a reformulation of a very particular case of Whitney's theorem. For $x \in \mathbb{R}^{n}$ and for a $C^{2}$-function $F$ defined in a neighborhood of $x$, we write $J_{x}(F)$ for the 2-jet of $F$ at $x$. That is, $J_{x}(F): \mathbb{R}^{2} \rightarrow \mathbb{R}$ is the Taylor polynomial of order two of $F$ about $x$.

Lemma 2.1 Let $\mathrm{U} \subseteq \mathbb{R}^{2}$ be an open set, and let $\mathrm{K} \subset \mathrm{U}$ be a bounded convex set whose closure $\overline{\mathrm{K}}$ is contained in $\mathrm{U}$. Let $\mathrm{M}>0$ and assume that $\mathrm{f}: \mathrm{U} \rightarrow \mathbb{R}$ is a $\mathrm{C}^{2}$-function with

$$
\|f\|_{C^{2}(u)} \leq M
$$

Then, there exists a $\mathrm{C}^{2}$-function $\mathrm{F}: \mathbb{R}^{2} \rightarrow \mathbb{R}$ with $\mathrm{F}(\mathrm{x})=\mathrm{f}(\mathrm{x})$ for all $\mathrm{x} \in \mathrm{K}$, such that

$$
\|\mathrm{F}\|_{\mathrm{C}^{2}} \leq \mathrm{C}_{W} \mathrm{M}
$$

Here, $\mathrm{C}_{\mathrm{W}}>1$ is a universal constant.

Proof. The functions $\partial^{x x_{f}}, \partial^{x y_{f}}, \partial^{y_{f}} f$ are continuous on the compact set $\bar{K}$. Let $\omega(\delta)(\delta>0)$ be the common modulus of continuity of those three functions on $\overline{\mathrm{K}}$. Fix $x, y \in \overline{\mathrm{K}}$. By applying Taylor's theorem for the interval $[x, y] \subseteq \overline{\mathrm{K}}$ we see that,

1. $\left|\partial^{\alpha}\left(J_{x}(f)-J_{y}(f)\right)(x)\right| \leq C M|x-y|^{2-|\alpha|}$ for $0 \leq|\alpha| \leq 2$,

2. $\left|\partial^{\alpha} f(x)-\partial^{\alpha} f(y)\right| \leq \omega(|x-y|)$ when $|\alpha|=2$ and $|x-y| \leq 1$.

3. $\left|\partial^{\alpha} f(x)\right| \leq C M$ for $0 \leq|\alpha| \leq 2$.

These are precisely the assumptions of the classical Whitney theorem (see [27] or [26, Section VI]). By the conclusion of that theorem, there exists a $C^{2}$-function $F: \mathbb{R}^{2} \rightarrow \mathbb{R}$ such that

1. $F(x)=f(x)$ for all $x \in K$,

2. $\|\mathrm{F}\|_{\mathrm{C}^{2}} \leq \mathrm{C}^{\prime} \mathrm{M}$.

Whitney's theorem also yields information regarding the modulus of continuity of the second derivatives of $F$; we do not use this information. The function $\mathrm{F}$ is our desired function. The proof is complete.

Whenever we write $C_{W}$ in this note, we always refer to the universal constant from Lemma 2.1. We will make use of the following three elementary lemmas. 
Lemma 2.2 Let $\mathrm{t}_{0}, \mathrm{t}_{1}, \mathrm{x}_{0}, \mathrm{x}_{0}^{\prime}, \mathrm{x}_{1}, \mathrm{x}_{1}^{\prime}$ be real numbers such that $\mathrm{t}_{0}<\mathrm{t}_{1}$, and let $\mathrm{M}>0$. Then the following are equivalent:

(a) $\left|\frac{\left(x_{1}-x_{0}\right)-\delta\left(x_{0}^{\prime}+x_{1}^{\prime}\right) / 2}{\delta^{2}}\right|<\frac{M}{4}\left[1-\left(\frac{x_{1}^{\prime}-x_{0}^{\prime}}{M \delta}\right)^{2}\right]$, where $\delta=t_{1}-t_{0}$.

(b) There exists a $C^{2}$-function $x: \mathbb{R} \rightarrow \mathbb{R}$ with $\sup _{\mathfrak{t} \in \mathbb{R}}\left|\chi^{\prime \prime}(\mathrm{t})\right|<M$ such that (2.1) $x\left(t_{0}\right)=x_{0}, x^{\prime}\left(t_{0}\right)=x_{0}^{\prime}, x^{\prime \prime}\left(t_{0}\right)=0 \quad x\left(t_{1}\right)=x_{1}, x^{\prime}\left(t_{1}\right)=x_{1}^{\prime}, x^{\prime \prime}\left(t_{1}\right)=0$.

Proof. By translating and rescaling in the $t$-axis, we may assume that $t_{0}=0, t_{1}=1$ and hence $\delta=1$. By rescaling in the $x$-axis, we may also assume that $M=1$. By adding an appropriate affine function, we may assume that $x_{0}=x_{0}^{\prime}=0$. Let $x(t)$ be a $C^{2}$-function such that (2.1) holds. Then,

$$
\int_{0}^{1} x^{\prime \prime}(\mathrm{t}) \mathrm{dt}=x_{1}^{\prime}, \quad \int_{0}^{1} x^{\prime \prime}(\mathrm{t}) \mathrm{tdt}=x_{1}^{\prime}-x_{1}
$$

as may be verified by integration by parts. Fix $\lambda \in(-1,1)$. We would like to understand the convex set

$$
\begin{gathered}
I_{\lambda}=\left\{\int_{0}^{1} u(t) t d t ;\right. \\
\int_{0}^{1} u(t) d t=\lambda, u(0)=u(1)=0, \\
\sup |u|<1, u \text { is continuous }\} .
\end{gathered}
$$

Note that $I_{\lambda}$ is the set of all possible values of $x^{\prime}(1)-x(1)$ where $x(t)$ is a $C^{2}$-function on $[0,1]$, with $x^{\prime}(1)=\lambda, x(0)=x^{\prime}(0)=x^{\prime \prime}(0)=x^{\prime \prime}(1)=0$ and $\sup \left|\chi^{\prime \prime}\right|<1$.

Suppose that $u:[0,1] \rightarrow \mathbb{R}$ is a continuous function with $u(0)=u(1)=0$ that satisfies $\sup |u|<1$ and $\int_{0}^{1} u(t) d t=\lambda$. Then,

$$
\begin{aligned}
\int_{0}^{1} u(t) t d t & =\frac{\lambda(1-\lambda)}{2}+\int_{0}^{1} u(t)\left(t-\frac{1-\lambda}{2}\right) d t \\
& <\frac{\lambda(1-\lambda)}{2}-\int_{0}^{(1-\lambda) / 2}\left(t-\frac{1-\lambda}{2}\right) d t+\int_{(1-\lambda) / 2}^{1}\left(t-\frac{1-\lambda}{2}\right) d t \\
(2.3) \quad & =\frac{\lambda}{2}+\frac{1-\lambda^{2}}{4} .
\end{aligned}
$$

Additionally, it is easy to find an admissible function $u$ for which equality almost holds in (2.3). This elementary line of reasoning leads to the conclusion that

$$
\mathrm{I}_{\lambda}=\left\{\mathrm{t} \in \mathbb{R} ; \frac{\lambda}{2}-\frac{1-\lambda^{2}}{4}<\mathrm{t}<\frac{\lambda}{2}+\frac{1-\lambda^{2}}{4}\right\} .
$$


Equivalently, there exists a $C^{2}$-function $x(t)$ with $\sup \left|x^{\prime \prime}\right|<1$ that satisfies (2.1) if and only if

$$
\left|x_{1}-x_{1}^{\prime} / 2\right|<\frac{1}{4}\left[1-\left(x_{1}^{\prime}\right)^{2}\right] .
$$

For a function $f:[a, b] \rightarrow \mathbb{R}$ we denote by $f^{\prime}(a), f^{\prime \prime}(b)$ etc. the corresponding one-sided derivatives. Thus, the notion of a $C^{2}$-function on a closed interval in $\mathbb{R}$ is well-defined. The proof of the following elementary lemma is very similar to that of Lemma 2.2 and it is omitted.

Lemma 2.3 Let $\mathrm{x}<\mathrm{y}$ be real numbers. Suppose $\mathrm{F}:[\mathrm{x}, \mathrm{y}] \rightarrow \mathbb{R}$ is a $\mathrm{C}^{1}$ function, that is also piecewise $\mathrm{C}^{2}$-smooth. Let $\mathrm{M}>0$, and assume that $\left|\mathrm{F}^{\prime \prime}(\mathrm{t})\right| \leq \mathrm{M}$ whenever the second derivative exists. Then,

$$
\left|\frac{(F(y)-F(x))-\delta\left(F^{\prime}(x)+F^{\prime}(y)\right) / 2}{\delta^{2}}\right| \leq \frac{M}{4}\left[1-\left(\frac{F^{\prime}(y)-F^{\prime}(x)}{M \delta}\right)^{2}\right],
$$

where $\delta=y-x$.

Our next lemma is yet another variant of Lemma 2.2, in which we force the first derivative to vanish on a certain set.

Lemma 2.4 Let $\mathrm{t}_{0}<\mathrm{t}_{1}, \mathrm{x}_{0}<\mathrm{x}_{1}, \varepsilon>0$ and let $\mathrm{t}_{\mathrm{0}}=\mathrm{p}_{1}<\mathrm{p}_{2}<\ldots<\mathrm{p}_{\mathrm{k}}=\mathrm{t}_{1}$ be a sequence of points. Assume that $\varepsilon \mathrm{k}<1 / 10$. Then, there exists a $\mathrm{C}^{2}$ function $\mathrm{x}:\left[\mathrm{t}_{0}, \mathrm{t}_{1}\right] \rightarrow \mathbb{R}$ with the following properties:

(a) $x\left(t_{0}\right)=x_{0}, x\left(t_{1}\right)=x_{1}$. Moreover, $x_{0} \leq x(t) \leq x_{1}$ for all $t \in\left[t_{0}, t_{1}\right]$.

(b) For any $i=1, \ldots, k$,

$$
x^{\prime}(t)=0 \quad \text { for } t \in\left[p_{i}-\varepsilon\left(t_{1}-t_{0}\right), p_{i}+\varepsilon\left(t_{1}-t_{0}\right)\right] \cap\left[t_{0}, t_{1}\right] .
$$

(c) $\left|x^{\prime}(t)\right| \leq(1+10 k \varepsilon)\left(x_{1}-x_{0}\right) /\left(t_{1}-t_{0}\right)$ for all $t \in\left[t_{0}, t_{1}\right]$.

(d) $\left|x^{\prime \prime}(t)\right| \leq 5 \varepsilon^{-1}\left(x_{1}-x_{0}\right) /\left(t_{1}-t_{0}\right)^{2}$ for all $t \in\left[t_{0}, t_{1}\right]$.

Proof. By translating and rescaling in the $t$-axis, we may assume that $t_{0}=0, t_{1}=1$. By translating and rescaling in the $x$-axis, we may also assume that $x_{0}=0$ and $x_{1}=1$. Consider the closed set

$$
A=\bigcup_{i=1}^{k}\left[p_{i}-2 \varepsilon, p_{i}+2 \varepsilon\right] \subseteq[-2 \varepsilon, 1+2 \varepsilon] .
$$


Then $A$ is a finite union of intervals containing $[-2 \varepsilon, 0] \cup[1,1+2 \varepsilon]$, and

$$
\kappa:=\operatorname{Meas}(A \cap[0,1]) \leq 4 k \varepsilon \leq 1 / 2 .
$$

Denote the interval components of $A$ by $I_{1}, \ldots, I_{\ell}$. Each of these intervals has length at least $4 \varepsilon$. We will construct a $C^{1}$-function $u(t)=x^{\prime}(t)$ on $[-2 \varepsilon, 1+2 \varepsilon]$ as follows: First, for any $t \in[-2 \varepsilon, 1+2 \varepsilon]$

$$
u(t)=\frac{1}{1-k+(\ell-1) \varepsilon} \quad \text { when } t \notin A \text {. }
$$

It remains to construct $u$ on the intervals $I_{1}, \ldots, I_{\ell}$. In order for the function to be $C^{1}$, we will force its derivative to vanish on the endpoints of the intervals $I_{1}, \ldots, I_{\ell}$. Fix an index $1 \leq i \leq \ell$, and write $I_{i}=\left[a_{i}, b_{i}\right]$. We then set

$$
u(t)=0 \quad \text { for } a_{i}+\varepsilon \leq t \leq b_{i}-\varepsilon .
$$

On the interval $\left[a_{i}, a_{i}+\varepsilon\right]$ we define $u$ to be

$$
u(t)=\frac{1}{1-\kappa+(\ell-1) \varepsilon}\left[\cos \left(\pi \frac{t-a_{i}}{\varepsilon}\right)+1\right] / 2 \quad\left(\text { for } a_{i} \leq t \leq a_{i}+\varepsilon\right) .
$$

Note that $u\left(a_{i}\right)=\frac{1}{1-\kappa+(\ell-1) \varepsilon}, u\left(a_{i}+\varepsilon\right)=0$ and $u^{\prime}\left(a_{i}\right)=u^{\prime}\left(a_{i}+\varepsilon\right)=0$. On the interval $\left[b_{i}-\varepsilon, b_{i}\right]$ we analogously define $u$ as

$$
u(t)=\frac{1}{1-k+(\ell-1) \varepsilon}\left[\cos \left(\pi \frac{b_{i}-t}{\varepsilon}\right)+1\right] / 2 \quad\left(\text { for } b_{i}-\varepsilon \leq t \leq b_{i}\right) .
$$

Then $u\left(b_{i}\right)=\frac{1}{1-\kappa+(\ell-1) \varepsilon}, u\left(b_{i}-\varepsilon\right)=0$ and $u^{\prime}\left(b_{i}\right)=u^{\prime}\left(b_{i}-\varepsilon\right)=0$. Consequently, the function $u$ is $C^{1}$-smooth on $\left[a_{i}, b_{i}\right]$ with

$$
0 \leq u(t) \leq \frac{1}{1-k+(\ell-1) \varepsilon}, \quad\left|u^{\prime}(t)\right| \leq \frac{\pi /(2 \varepsilon)}{1-\kappa+(\ell-1) \varepsilon}
$$

for $a_{i} \leq t \leq b_{i}$. In addition,

$$
\varepsilon^{-1} \int_{a_{i}}^{a_{i}+\varepsilon} u(t) d t=\varepsilon^{-1} \int_{b_{i}-\varepsilon}^{b_{i}} u(t) d t=\frac{1}{2(1-k+(\ell-1) \varepsilon)} .
$$

This completes the definition of the function $u:[-2 \varepsilon, 1+2 \varepsilon] \rightarrow \mathbb{R}$. The function $u$ is continuous, since it attains the right values at the endpoints of $I_{1}, \ldots, I_{\ell}$. Moreover, $u$ is $C^{1}$-smooth, since it is $C^{1}$-smooth in $A$, constant on the complement of $A$, and its derivative vanishes at the endpoints of the 
interval components of $A$. By the construction, we clearly have that for $i=1, \ldots, k$,

$$
u(t)=0 \quad \text { when } t \in\left[p_{i}-\varepsilon, p_{i}+\varepsilon\right] \cap[0,1] .
$$

Note that $(1-\kappa+(\ell-1) \varepsilon)^{-1} \leq(1-\kappa)^{-1} \leq 1+10 k \varepsilon \leq 2$. Thus (2.6) implies that

$$
0 \leq u(t) \leq 1+10 k \varepsilon, \quad\left|u^{\prime}(t)\right| \leq \frac{5}{\varepsilon} \quad \text { for } \quad 0 \leq t \leq 1 .
$$

Moreover, by (2.4), (2.5) and (2.7),

$$
\begin{aligned}
\int_{-2 \varepsilon}^{1+2 \varepsilon} u(t) d t & =\frac{M \operatorname{eas}([-2 \varepsilon, 1+2 \varepsilon] \backslash A)}{1-\kappa+(\ell-1) \varepsilon}+\sum_{i=1}^{\ell} \int_{a_{i}}^{b_{i}} u(t) d t \\
& =\frac{1-\kappa}{1-\kappa+(\ell-1) \varepsilon}+\ell \cdot 2 \cdot \frac{\varepsilon}{2(1-\kappa+(\ell-1) \varepsilon)} \\
& =1+\frac{\varepsilon}{1-\kappa+(\ell-1) \varepsilon}
\end{aligned}
$$

However, one of the interval components of $A$ begins with $-2 \varepsilon$, and additionally, one of the interval components of $A$ ends with $1+2 \varepsilon$, since $p_{0}=0, p_{k}=1$. Consequently, by using (2.10), (2.5) and (2.7),

$$
\int_{0}^{1} u(t) d t=1+\frac{\varepsilon}{1-\kappa+(\ell-1) \varepsilon}-\left[\int_{-2 \varepsilon}^{0} u(t) d t+\int_{1}^{1+2 \varepsilon} u(t) d t\right]=1
$$

Denote $x(t)=\int_{0}^{t} u(s) d s$. Since $u$ is $C^{1}$-smooth, then $x$ is $C^{2}$-smooth. Moreover, $x(0)=0$ and $x(1)=1$, according to (2.11). The fact that $u \geq 0$ (see $(2.9)$ ) implies that $0 \leq x(t) \leq 1$ for all $t \in[0,1]$. Hence $x$ satisfies (a). From (2.8) we see that (b) holds, while (c), (d) hold in view of (2.9). The proof of the lemma is complete.

\section{The Construction}

Suppose we are given a positive integer $k^{\#}>0$. In this section, we construct a certain finite set $E \subset \mathbb{R}^{2}$ and a function $f: E \rightarrow \mathbb{R}$. The construction depends on $k^{\#}$. In later sections, we will verify that $E$ and $f$ satisfy the assertions of Proposition 1.3.

We begin by defining the small scales $\varepsilon, \delta>0$, with $\delta$ being much smaller than $\varepsilon$. We first define $\varepsilon=\left(2 k^{\#}\right)^{-10}$, so that $1 / \varepsilon$ is an even integer. Then we select $\delta=\varepsilon^{20}$. Note that $1 / \delta, \varepsilon / \delta$ and $\varepsilon^{2} / \delta$ are all integers. 
Define

$$
\ell_{1}=\left\{(\delta i, 0) ; i=0, \ldots, \delta^{-1} \varepsilon\right\} \subset \mathbb{R}^{2}, \quad \ell_{2}=\{(0, \delta),(\varepsilon, \delta)\} \subset \mathbb{R}^{2}
$$

Our set $E$ will be simply $E=\ell_{1} \cup \ell_{2}$; see Figure 2 .

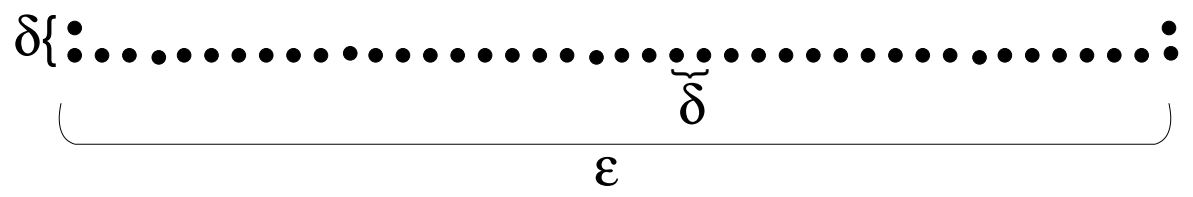

Figure 2

Define a function zigzag $: \mathbb{R} \rightarrow \mathbb{R}$ as follows:

$$
\operatorname{zigzag}(x)=\left\{\begin{array}{cc}
-\frac{\{x\}}{4}+\frac{\{x\}^{2}}{2} & \{x\} \leq \frac{1}{2} \\
-\frac{1}{4}+\frac{3}{4}\{x\}-\frac{\{x\}^{2}}{2} & \{x\}>\frac{1}{2}
\end{array}\right.
$$

Here, $\{x\}=x-\lfloor x\rfloor$ is the fractional part of $x$. The function zigzag is a 1 -periodic $C^{1}$-function, and whenever $2 x \notin \mathbb{Z}$,

$$
|\operatorname{zigzag}(x)| \leq \frac{1}{32}, \quad\left|\operatorname{zigzag}^{\prime}(x)\right| \leq \frac{1}{4}, \quad \text { and } \quad\left|\operatorname{zigzag}^{\prime \prime}(x)\right|=1
$$

The function $z \operatorname{igzag}(x)$ goes back and forth between $-\frac{1}{4}$ and $\frac{1}{4}$, with constant speed one. Hence the term "zigzag".

Next, we define two functions on $\mathbb{R}$ :

$$
f_{1}(x)=C_{W}^{\prime}\left(\frac{\delta}{\varepsilon}\right)^{2} \text { zigzag }\left(\frac{\varepsilon x}{\delta}\right), \quad f_{2}(x)=x /\left(2 C_{W}\right)
$$

where $C_{W}^{\prime}=1-10^{-6} / C_{W}^{4}$ is a universal constant that is just slightly smaller than one. Throughout this text, we write $\mathrm{C}_{W}^{\prime}$ to denote the constant $1-10^{-6} / C_{W}^{4}$. Finally, we set $f: E \rightarrow \mathbb{R}$ to be

$$
f(x, y)=f_{1}(x)+y f_{2}(x) \quad \text { for }(x, y) \in E
$$

This completes the description of the set $E \subset \mathbb{R}^{2}$ and the function $f: E \rightarrow \mathbb{R}$. 


\section{Any extension has a large $C^{2}$ norm}

Suppose we are given an integer $k^{\#}$. Throughout this section, let $E$ and $f, f_{1}, f_{2}$, zigzag be the finite set and the functions constructed in the previous section for $k^{\#}$. We will prove the following lemma.

Lemma 4.1 Suppose that $\mathrm{k}^{\#}>\mathrm{C}$, and assume that $\mathrm{F}: \mathbb{R}^{2} \rightarrow \mathbb{R}$ is a $\mathrm{C}^{2}$ smooth function that satisfies $\left.\mathrm{F}\right|_{\mathrm{E}}=\mathrm{f}$. Then,

$$
\|\mathrm{F}\|_{C^{2}}>1+10^{-4} / C_{W}^{3}
$$

where $\mathrm{C}_{\mathrm{W}}>0$ is the universal constant from Lemma 2.1, and where $\mathrm{C}>0$ is a universal constant.

The proof of Lemma 4.1 relies on several lemmas.

Lemma 4.2 Suppose that $\mathrm{k}^{\#}>\mathrm{C}$, and assume that $\mathrm{F}: \mathbb{R}^{2} \rightarrow \mathbb{R}$ is a $\mathrm{C}^{2}$ smooth function with $\left.\mathrm{F}\right|_{\mathrm{E}}=\mathrm{f}$ such that

$$
\|\mathrm{F}\|_{\mathrm{C}^{2}} \leq 1+10^{-4} / \mathrm{C}_{W}^{3}
$$

Then there exists a subset $A \subseteq[0, \varepsilon]$, whose Lebesgue measure is at least $\left(1-\frac{1}{10 C_{w}}\right) \cdot \varepsilon$, such that

$$
\left|\partial^{x x} F(t, 0)\right|>1-\frac{1}{10 C_{W}^{2}} \text { for all } t \in A .
$$

Here, $\mathrm{C}>0$ is a universal constant.

Proof. By choosing a sufficiently large constant $C>0$, we may assume that $k^{\#}>100 C_{W}$ and hence $\varepsilon<10^{-5} / C_{W}^{10}$ and $\delta / \varepsilon^{2}<1 /\left(50 C_{W}\right)$. The disjoint union

$$
\bigcup_{i=0}^{\varepsilon^{2} / \delta-1}\left[i \frac{\delta}{\varepsilon},(i+1) \frac{\delta}{\varepsilon}\right)
$$

covers the entire interval $[0, \varepsilon)$. Thus, to establish the lemma, it is sufficient to show that for any $0 \leq i \leq \frac{\varepsilon^{2}}{\delta}-1$,

$$
\text { Meas }\left\{t \in\left[i \frac{\delta}{\varepsilon},(i+1) \frac{\delta}{\varepsilon}\right) ;\left|\partial^{x x} F(t, 0)\right|>1-\frac{1}{10 C_{W}^{2}}\right\} \geq\left(1-\frac{1}{10 C_{W}}\right) \cdot \frac{\delta}{\varepsilon} \text {. }
$$

We will begin by showing that for any $i$,

$$
\text { Meas }\left\{t \in\left[i \frac{\delta}{\varepsilon},\left(i+\frac{1}{2}\right) \frac{\delta}{\varepsilon}\right) ; \partial^{x x} F(t, 0) \leq 1-\frac{1}{10 C_{W}^{2}}\right\}<\frac{1}{10 C_{W}} \cdot \frac{\delta}{2 \varepsilon} \text {. }
$$


Fix an integer $0 \leq i \leq \frac{\varepsilon^{2}}{\delta}-1$. Then there exist two distinct points $\left(p_{1}, 0\right),\left(p_{2}, 0\right) \in \ell_{1}$ such that

$$
i \frac{\delta}{\varepsilon}<p_{1}<p_{2} \leq i \frac{\delta}{\varepsilon}+2 \delta
$$

Since $\left.F\right|_{E}=f$, then

$$
\begin{aligned}
\frac{F\left(p_{2}, 0\right)-F\left(p_{1}, 0\right)}{p_{2}-p_{1}} & =\frac{f\left(p_{2}, 0\right)-f\left(p_{1}, 0\right)}{p_{2}-p_{1}}=\frac{f_{1}\left(p_{2}\right)-f_{1}\left(p_{1}\right)}{p_{2}-p_{1}} \\
& \leq C_{W}^{\prime} \sup _{p_{1} \leq t \leq p_{2}}\left(\frac{\delta}{\varepsilon}\right) z i g z a g^{\prime}\left(\frac{\varepsilon}{\delta} t\right) \\
& \leq C_{W}^{\prime}{ }^{\frac{\delta}{\varepsilon}} \sup _{i \leq t \leq i+2 \varepsilon} z i g z a g^{\prime}(t) \leq \frac{\delta}{\varepsilon} C_{W}^{\prime} \cdot\left(-\frac{1}{4}+2 \varepsilon\right)
\end{aligned}
$$

By Lagrange's theorem, there exists a point $(p, 0)$ with $i \frac{\delta}{\varepsilon} \leq p \leq i \frac{\delta}{\varepsilon}+2 \delta$ such that

$$
\partial^{x} \mathrm{~F}(\mathrm{p}, 0) \leq \frac{\delta}{\varepsilon} \cdot \mathrm{C}_{W}^{\prime}\left(-\frac{1}{4}+2 \varepsilon\right)
$$

Similarly, there exists a point $(q, 0)$ with $(i+1 / 2) \frac{\delta}{\varepsilon}-2 \delta \leq q \leq(i+1 / 2) \frac{\delta}{\varepsilon}$ such that

$$
\partial^{x} F(q, 0) \geq \frac{\delta}{\varepsilon} \cdot C_{W}^{\prime}\left(\frac{1}{4}-2 \varepsilon\right) \text {. }
$$

Consequently,

$$
\frac{C_{W}^{\prime}}{q-p} \cdot \frac{\delta}{\varepsilon}\left(\frac{1}{2}-4 \varepsilon\right) \leq \frac{\partial^{x} F(q, 0)-\partial^{x} F(p, 0)}{q-p}=\frac{1}{q-p} \int_{p}^{q} \partial^{x x} F(t, 0) d t
$$

However, $q-p \leq \frac{\delta}{2 \varepsilon}$. Hence,

$$
\begin{aligned}
\frac{C_{W}^{\prime}}{q-p} \cdot \frac{\delta}{\varepsilon}\left(\frac{1}{2}-4 \varepsilon\right) & \geq C_{W}^{\prime}(1-8 \varepsilon)=\left(1-10^{-6} / C_{W}^{4}\right) \cdot(1-8 \varepsilon) \\
& >1-10^{-3} / C_{W}^{3}
\end{aligned}
$$

since $\varepsilon<10^{-5} \mathrm{C}_{W}^{-10}$. From (4.3) and (4.4) we obtain

$$
1-10^{-3} / C_{W}^{3}<\frac{1}{q-p} \int_{p}^{q} \partial^{x x} F(t, 0) d t
$$

Recall that $\partial^{x x} \mathrm{~F}(z) \leq\|\mathrm{F}\|_{\mathrm{C}^{2}} \leq 1+10^{-4} / \mathrm{C}_{W}^{3}$ for any $z \in \mathbb{R}^{2}$, according to our definition (1.3) of the $\mathrm{C}^{2}$ norm. We claim that

$$
\operatorname{Meas}\left\{t \in[p, q] ; \partial^{x x} F(t, 0) \leq 1-\frac{1}{10 C_{W}^{2}}\right\}<\frac{1}{30 C_{W}} \cdot(q-p) \text {. }
$$


Indeed, if (4.6) does not hold, then

$$
\begin{aligned}
\frac{1}{q-p} \int_{p}^{q} \partial^{x x} F(t, 0) d t & \leq \frac{1}{30 C_{W}}\left(1-\frac{1}{10 C_{W}^{2}}\right)+\left(1-\frac{1}{30 C_{W}}\right) \cdot\left(1+\frac{10^{-4}}{C_{W}^{3}}\right) \\
& <1-10^{-3} / C_{W}^{3},
\end{aligned}
$$

in contradiction to (4.5). This completes the proof of (4.6). The interval $[p, q]$ is a very good approximation to the interval $\left[i \frac{\delta}{\varepsilon},(i+1 / 2) \frac{\delta}{\varepsilon}\right]$; The measure of their symmetric difference does not exceed $4 \delta \leq \frac{\delta}{60 C_{W} \varepsilon}$. Therefore, (4.6) implies (4.2).

This completes the proof of (4.2). The proof that for any $0 \leq i \leq \varepsilon^{2} / \delta-1$, (4.7)

Meas $\left\{t \in\left[\left(i+\frac{1}{2}\right) \frac{\delta}{\varepsilon},(i+1) \frac{\delta}{\varepsilon}\right) ; \partial^{x x} F(t, 0) \geq-1+\frac{1}{10 C_{W}^{2}}\right\}<\frac{1}{10 C_{W}} \cdot \frac{\delta}{2 \varepsilon}$,

is completely analogous, and it is omitted. From (4.2) and (4.7) we deduce (4.1). The lemma follows.

Lemma 4.3 Suppose that $\mathrm{k}^{\#}>\mathrm{C}$, and assume that $\mathrm{F}: \mathbb{R}^{2} \rightarrow \mathbb{R}$ is a $\mathrm{C}^{2}$ smooth function with $\left.\mathrm{F}\right|_{\mathrm{E}}=\mathrm{f}$ such that

$$
\|\mathrm{F}\|_{\mathrm{C}^{2}} \leq 1+10^{-4} / \mathrm{C}_{W}^{3}
$$

Then there exists a subset $A \subseteq[0, \varepsilon]$, whose Lebesgue measure is at least $\frac{\varepsilon}{9 C_{W}}$, such that

$$
\partial^{x y} \mathrm{~F}(\mathrm{t}, 0)>\frac{1}{3 \mathrm{C}_{W}} \quad \text { for all } \mathrm{t} \in \mathrm{A} .
$$

Here, $\mathrm{C}>0$ is a universal constant.

Proof. Fix $i=0,1$. Then both points $(i \varepsilon, 0)$ and $(i \varepsilon, \delta)$ belong to the finite set $E$. Since $\left.F\right|_{E}=f$, then $F(i \varepsilon, \delta)-F(i \varepsilon, 0)=\delta f_{2}(i \varepsilon)$. By Lagrange's theorem, $\partial^{y} F(i \varepsilon, \theta \delta)=f_{2}(i \varepsilon)$ for some $0 \leq \theta \leq 1$. Since $\|F\|_{C^{2}} \leq 1+$ $10^{-4} / C_{W}^{2} \leq 2$, then $\left|\partial^{y y} \mathrm{~F}(z)\right| \leq 2$ for all $z \in \mathbb{R}^{2}$, by (1.3). Hence,

$$
\left|\partial^{y} F(i \varepsilon, 0)-f_{2}(i \varepsilon)\right| \leq 2 \delta \text { for } i=0,1 \text {. }
$$

However,

$$
f_{2}(0)=0 \quad \text { and } \quad f_{2}(\varepsilon)=\varepsilon /\left(2 C_{W}\right) .
$$

We assume that $\mathrm{k}^{\#}$ exceeds a certain universal constant, so that $\delta<\varepsilon^{2} /\left(8 \mathrm{C}_{W}\right)$ and $\varepsilon<1 / 10$. Then (4.8) and (4.9) imply

$$
\frac{1-\varepsilon}{2 C_{W}} \leq \frac{\partial^{y} F(\varepsilon, 0)-\partial^{y} F(0,0)}{\varepsilon}=\frac{1}{\varepsilon} \int_{0}^{\varepsilon} \partial^{x y} F(t, 0) d t .
$$


By our definition (1.3) of the $C^{2}$ norm, $2\left(\partial^{x y} F\right)^{2} \leq\|F\|_{C^{2}}^{2}$. Since $\|F\|_{C^{2}}^{2} \leq$ $1+10^{-3} \leq 2$, we have that $\left|\partial^{x y} \mathrm{~F}(z)\right| \leq 1$ for all $z \in \mathbb{R}^{2}$. To prove the lemma, we need to show that

$$
\text { Meas }\left\{t \in[0, \varepsilon] ; \partial^{x y} F(t, 0)>\frac{1}{3 C_{W}}\right\} \geq \frac{\varepsilon}{9 C_{W}} \text {. }
$$

Assume by contradiction that $\partial^{x y} F(z)>\frac{1}{3 C_{W}}$ for at most $\frac{1}{9 C_{W}}$-portion of the interval $[0, \varepsilon] \times\{0\}$. Then,

$$
\frac{1}{\varepsilon} \int_{0}^{\varepsilon} \partial^{x y} F(t, 0) d t \leq\left(1-\frac{1}{9 C_{W}}\right) \cdot \frac{1}{3 C_{W}}+\frac{1}{9 C_{W}} \cdot 1 \leq \frac{4}{9 C_{W}}<\frac{1-\varepsilon}{2 C_{W}}
$$

as $\varepsilon<1 / 10$, in contradiction to (4.10). Thus (4.11) is proved. The lemma follows.

Proof of Lemma 4.1. Assume, by contradiction, that $F: \mathbb{R}^{2} \rightarrow \mathbb{R}$ is a $\mathrm{C}^{2}$-smooth function with $\left.\mathrm{F}\right|_{\mathrm{E}}=\mathrm{f}$ such that

$$
\|\mathrm{F}\|_{\mathrm{C}^{2}} \leq 1+10^{-4} / \mathrm{C}_{W}^{3}
$$

We combine Lemma 4.2 and Lemma 4.3 to conclude that there is a point $t \in[0, \varepsilon]$ such that

$$
\left|\partial^{x y} F(t, 0)\right| \geq \frac{1}{3 C_{W}}, \quad \text { and } \quad\left|\partial^{x x} F(t, 0)\right| \geq 1-\frac{1}{10 C_{W}^{2}}
$$

(actually, at least $\frac{1}{90 C_{w}}$-portion of $[0, \varepsilon]$ have this property). Consequently, from our definition (1.3) of the $\mathrm{C}^{2}$ norm,

$$
\|F\|_{C^{2}}^{2} \geq\left|\partial^{x x} F(t, 0)\right|^{2}+2\left|\partial^{x y} F(t, 0)\right|^{2} \geq\left(1-\frac{1}{10 C_{W}^{2}}\right)^{2}+\frac{2}{9 C_{W}^{2}} \geq 1+\frac{1}{45 C_{W}^{2}},
$$

in contradiction to our assumption (4.12) that $\|\mathrm{F}\|_{C^{2}} \leq 1+10^{-4} / C_{W}^{3}$.

\section{Functions that agree with $f$ on $k^{\#}$ points}

Suppose we are given an integer $k^{\#}$. Throughout this section, let $E$ and $f, f_{1}, f_{2}$ be the finite set and the functions constructed in Section 3 for $k^{\#}$. In this section, we will construct a $C^{2}$-function on $\mathbb{R}^{2}$, with a small $C^{2}$ norm, that agrees with $f$ on given $k^{\#}$ points of $E$.

Thus, suppose we are given a subset $S \subseteq E$ whose cardinality is bounded by $k^{\#}$. Let $T=\{x ;(x, y) \in S\} \cup\{0, \varepsilon\}$. 
Lemma 5.1 Suppose that $\mathrm{k}^{\#}>\mathrm{C}$. Then there exists a $\mathrm{C}^{2}$-smooth function $\mathrm{F}: \mathbb{R}^{2} \rightarrow \mathbb{R}$ with the following properties:

1. $F(x, 0)=f(x, 0)$ for all $x \in T$,

2. $F(0, \delta)=f(0, \delta)$ and $F(\varepsilon, \delta)=f(\varepsilon, \delta)$.

3. $\|\mathrm{F}\|_{C^{2}} \leq 1$.

Here, $\mathrm{C}>0$ is a universal constant.

Note that, in particular, a function $F$ as in Lemma 5.1 agrees with $f$ on the set $S$.

The construction of the desired function $F$ requires a few steps, and it is described in the following series of lemmas. Denote $k=\#(T) \leq k^{\#}+2$. Let $0=p_{1}<p_{2}<\ldots<p_{k}=\varepsilon$ be an enumeration of the points of $\mathrm{T}$. Recall the definition of the set $\ell_{1}$ and the function $f_{1}$ from Section 3. Recall that $\mathrm{C}_{\mathrm{W}}^{\prime}=1-10^{-6} / \mathrm{C}_{\mathrm{W}}^{4}$.

Lemma 5.2 Suppose that $\mathrm{k}^{\#}>\mathrm{C}$. Then there exists a $\mathrm{C}^{2}$-function $\mathrm{F}_{1}$ : $\mathbb{R} \rightarrow \mathbb{R}$ with the following properties:

(a) $\mathrm{F}_{1}\left(\mathrm{p}_{\mathrm{i}}\right)=\mathrm{f}_{1}\left(\mathrm{p}_{\mathrm{i}}\right)$ for $\mathrm{i}=1, \ldots, \mathrm{k}$.

(b) $\left|\mathrm{F}_{1}(\mathrm{t})\right|<5 \delta / \varepsilon^{2},\left|\mathrm{~F}_{1}^{\prime}(\mathrm{t})\right|<5 \delta / \varepsilon^{2}$ and $\left|\mathrm{F}_{1}^{\prime \prime}(\mathrm{t})\right| \leq \mathrm{C}_{W}^{\prime}+\varepsilon$ for all $\mathrm{t} \in \mathbb{R}$.

(c) $\left|\mathrm{F}_{1}^{\prime \prime}(\mathrm{t})\right|<\varepsilon$ whenever $\mathrm{t} \in\left[p_{i-1}, p_{i}\right]$ with $p_{i}-p_{i-1} \geq 2 \delta / \varepsilon^{2}$ for $i=$ $2, \ldots, k$.

(d) $\left|\mathrm{F}_{1}^{\prime \prime}(\mathrm{t})\right|<\varepsilon$ if $\mathrm{t}<0$ or $\mathrm{t}>\varepsilon$.

Proof. The function $f_{1}$ is $C^{1}$-smooth and piecewise $C^{2}$-smooth. For any $t \in[0, \varepsilon]$, except for finitely many points, we have that

$$
\left|f_{1}(t)\right| \leq \frac{1}{32} \cdot\left(\frac{\delta}{\varepsilon}\right)^{2},\left|f_{1}^{\prime}(t)\right| \leq \frac{\delta}{4 \varepsilon} \text { and }\left|f_{1}^{\prime \prime}(t)\right| \leq C_{W}^{\prime} .
$$

(we used the fact that $C_{W}^{\prime} \leq 1$.) Fix $2 \leq i \leq k$. We begin by constructing a $\mathrm{C}^{2}$-function $\mathrm{F}_{1}^{\mathrm{i}}:\left[\mathrm{p}_{\mathrm{i}-1}, \mathrm{p}_{i}\right] \rightarrow \mathbb{R}$ such that

$$
F_{1}^{i}\left(p_{j}\right)=f_{1}\left(p_{j}\right),\left(F_{1}^{i}\right)^{\prime}\left(p_{j}\right)=f_{1}^{\prime}\left(p_{j}\right),\left(F_{1}^{i}\right)^{\prime \prime}\left(p_{j}\right)=0 \quad \text { for } j=i-1, i .
$$

Later on, we will define $F_{1}$ so that it will agree with $F_{1}^{i}$ on the interval $\left[p_{i-1}, p_{i}\right]$. The definition of $F_{1}^{i}$ is divided into two cases. 
Case 1: Suppose $p_{i}-p_{i-1} \geq 2 \delta / \varepsilon^{2}$. Let $M>0$ be such that $p_{i}-p_{i-1}=$ $2 \delta /(\varepsilon M)$. Then $M \leq \varepsilon$, and by $(5.1)$,

$$
\begin{gathered}
\left|\frac{\left(f_{1}\left(p_{i}\right)-f_{1}\left(p_{i-1}\right)\right)-\left(p_{i}-p_{i-1}\right) \cdot\left[f_{1}^{\prime}\left(p_{i-1}\right)+f_{1}^{\prime}\left(p_{i}\right)\right] / 2}{\left(p_{i}-p_{i-1}\right)^{2}}\right| \\
\leq \frac{(2 / 32) \cdot(\delta / \varepsilon)^{2}}{(2 \delta /(\varepsilon M))^{2}}+\frac{\delta /(4 \varepsilon)}{2 \delta /(\varepsilon M)}<\frac{M}{4}\left(1-\frac{1}{4}\right) \\
<\frac{M}{4}\left[1-\left(\frac{f_{1}^{\prime}\left(p_{i}\right)-f_{1}^{\prime}\left(p_{i-1}\right)}{M\left(p_{i}-p_{i-1}\right)}\right)^{2}\right] .
\end{gathered}
$$

By Lemma 2.2 we can construct a $C^{2}$-function $F_{1}^{i}:\left[p_{i-1}, p_{i}\right] \rightarrow \mathbb{R}$ such that $\left|\left(F_{1}^{i}\right)^{\prime \prime}(t)\right|<M \leq \varepsilon$ for $t \in\left[p_{i-1}, p_{i}\right]$, and such that (5.2) holds. Note that, thanks to (5.1) and (5.2), for $t \in\left[p_{i-1}, p_{i}\right]$,

$$
\begin{aligned}
\left|\left(F_{1}^{i}\right)^{\prime}(t)\right| & \leq\left|\left(F_{1}^{i}\right)^{\prime}\left(p_{i-1}\right)\right|+\left(p_{i}-p_{i-1}\right) \cdot \sup _{t \in\left[p_{i-1}, p_{i}\right]}\left|\left(F_{1}^{i}\right)^{\prime \prime}(t)\right| \\
& \leq \frac{\delta}{4 \varepsilon}+M\left(p_{i}-p_{i-1}\right)<\frac{3 \delta}{\varepsilon} .
\end{aligned}
$$

Additionally, for any $t \in\left[p_{i-1}, p_{i}\right]$,

$$
\left|F_{1}^{i}(t)\right| \leq\left|F_{1}^{i}(0)\right|+\left(p_{i}-p_{i-1}\right) \cdot \frac{3 \delta}{\varepsilon}<\frac{5 \delta}{\varepsilon} .
$$

To summarize, when $p_{i}-p_{i-1} \geq 2 \delta / \varepsilon^{2}$, the $C^{2}$-function $F_{1}^{i}:\left[p_{i-1}, p_{i}\right] \rightarrow \mathbb{R}$ satisfies (5.2) and also

$$
\left|F_{1}^{i}(t)\right|<5 \delta / \varepsilon,\left|\left(F_{1}^{i}\right)^{\prime}(t)\right|<5 \delta / \varepsilon \text { and }\left|\left(F_{1}^{i}\right)^{\prime \prime}(t)\right|<\varepsilon \text { for all } t \in\left[p_{i-1}, p_{i}\right] .
$$

Case 2: Suppose $p_{i}-p_{i-1}<2 \delta / \varepsilon^{2}$. The function $f_{1}$ is $C^{1}$-smooth and piecewise $C^{2}$-smooth on $\left[p_{i-1}, p_{i}\right]$. Moreover, its second derivative does not exceed $C_{W}^{\prime}$ in absolute value, whenever it exists, according to (5.1). By applying Lemma 2.3 and Lemma 2.2 we find that there exists a $C^{2}$-function $\mathrm{F}_{1}^{\mathrm{i}}:\left[\mathrm{p}_{\mathrm{i}-1}, \mathrm{p}_{\mathrm{i}}\right] \rightarrow \mathbb{R}$ such that

$$
\left|\left(F_{1}^{i}\right)^{\prime \prime}(t)\right|<C_{W}^{\prime}+\varepsilon \quad \text { for } \quad t \in\left[p_{i-1}, p_{i}\right]
$$

and such that (5.2) holds.

Since $\left|\left(F_{1}^{i}\right)^{\prime}\left(p_{i-1}\right)\right| \leq \delta /(4 \varepsilon)$ by $(5.1)$, then for any $t \in\left[p_{i-1}, p_{i}\right]$,

$$
\left|\left(F_{1}^{i}\right)^{\prime}(t)\right| \leq\left|\left(F_{1}^{i}\right)^{\prime}\left(p_{i-1}\right)\right|+\left(C_{W}^{\prime}+\varepsilon\right)\left(p_{i}-p_{i-1}\right) \leq \delta /(4 \varepsilon)+\frac{2 \delta}{\varepsilon^{2}}\left(C_{W}^{\prime}+\varepsilon\right) \leq \frac{4 \delta}{\varepsilon^{2}} .
$$

Similarly, we see that $\left|F_{1}^{i}(t)\right| \leq 10 \delta^{2} / \varepsilon^{4} \leq 5 \delta$ for $t \in\left[p_{i-1}, p_{i}\right]$. 
To summarize, when $p_{i}-p_{i-1}<2 \delta / \varepsilon^{2}$, the $C^{2}$-function $F_{1}^{i}:\left[p_{i-1}, p_{i}\right] \rightarrow \mathbb{R}$ satisfies (5.2) and also

$$
\begin{aligned}
& \left|\mathrm{F}_{1}^{\mathrm{i}}(\mathrm{t})\right|<5 \delta / \varepsilon^{2}, \quad\left|\left(\mathrm{~F}_{1}^{\mathrm{i}}\right)^{\prime}(\mathrm{t})\right|<5 \delta / \varepsilon^{2} \text { and } \\
& \quad\left|\left(\mathrm{F}_{1}^{\mathrm{i}}\right)^{\prime \prime}(\mathrm{t})\right| \leq \mathrm{C}_{W}^{\prime}+\varepsilon \text { for all } \mathrm{t} \in\left[\mathrm{p}_{\mathrm{i}-1}, \mathrm{p}_{\mathrm{i}}\right] .
\end{aligned}
$$

This completes the construction of the $C^{2}$-function $F_{1}^{i}:\left[p_{i-1}, p_{i}\right] \rightarrow \mathbb{R}$ in all cases. Note that $F_{1}^{i}$ satisfies (5.2) for all $2 \leq i \leq k$.

Next, we shall define two functions, in $[\varepsilon, 1]$ and in $[-1,0]$, as follows: We define $G:[\varepsilon, 1] \rightarrow \mathbb{R}$ to be a $C^{2}$-function such that $\left|G^{\prime \prime}(t)\right| \leq 5 \delta / \varepsilon$ for $t \in[\varepsilon, 1]$ and

$$
\mathrm{G}(\varepsilon)=\mathrm{f}_{1}(\varepsilon), \mathrm{G}^{\prime}(\varepsilon)=\mathrm{f}_{1}^{\prime}(\varepsilon), \quad \mathrm{G}^{\prime \prime}(\varepsilon)=\mathrm{G}(1)=\mathrm{G}^{\prime}(1)=\mathrm{G}^{\prime \prime}(1)=0 .
$$

The existence of such $\mathrm{G}$ is guaranteed by (5.1) and Lemma 2.2. Note that necessarily $\left|\mathrm{G}^{\prime}\right| \leq 10 \delta / \varepsilon$ and $|\mathrm{G}| \leq 20 \delta / \varepsilon$ on $[\varepsilon, 1]$.

Similarly, we define $H:[-1,0] \rightarrow \mathbb{R}$ to be a $C^{2}$-function with $\left|H^{\prime \prime}\right| \leq 5 \delta / \varepsilon$ such that

$$
\begin{aligned}
H(0) & =f_{1}(0), \\
H^{\prime}(0) & =f_{1}^{\prime}(0), \\
H^{\prime \prime}(0) & =H(-1)=H^{\prime}(-1)=H^{\prime \prime}(-1)=0 .
\end{aligned}
$$

The existence of such $\mathrm{H}$ is again guaranteed by (5.1) and Lemma 2.2, and again $\left|H^{\prime}\right| \leq 10 \delta / \varepsilon$ and $|H| \leq 20 \delta / \varepsilon$. Finally, we define

$$
F_{1}(t)=\left\{\begin{array}{cc}
0 & t \leq-1 \\
H(t) & -1<t<0 \\
F_{1}^{i}(t) & p_{i-1} \leq t \leq p_{i} \\
G(t) & \varepsilon<t<1 \\
0 & t \geq 1
\end{array}\right.
$$

The function $F_{1}$ is defined on the entire real line. Since $G, H, F_{1}^{i}(i=2, \ldots, k)$ are $\mathrm{C}^{2}$-smooth on their domain of definition, and since (5.2), (5.5) and (5.6) hold, then $F_{1}$ is $C^{2}$-smooth. Next, we verify the desired properties (a),.., (d) from the formulation of the lemma. Property (a) holds in view of (5.2). Property (c) follows from (5.3), while property (d) is evident from the definitions of $\mathrm{G}$ and H. Property (b) holds because of (5.3), (5.4) and the corresponding properties of $\mathrm{G}$ and $\mathrm{H}$. The proof is complete.

Recall from the beginning of this section the list of points $0=p_{1}<\ldots<$ $p_{k}=\varepsilon$. Recall also the function $f_{2}(t)=t /\left(2 C_{W}\right)$. 
Lemma 5.3 Suppose that $\mathrm{k}^{\#}>\mathrm{C}$. Then there exists a $\mathrm{C}^{2}$-function $\mathrm{F}_{2}$ : $\left[-\varepsilon^{2}, \varepsilon+\varepsilon^{2}\right] \rightarrow \mathbb{R}$ such that

(i) $\mathrm{F}_{2}(\varepsilon i)=\mathrm{f}_{2}(\varepsilon i)$ for $i=0,1$.

(ii) For all $\mathrm{t} \in\left[-\varepsilon^{2}, \varepsilon+\varepsilon^{2}\right]$,

$$
\left|\mathrm{F}_{2}(\mathrm{t})\right|<5 \varepsilon, \quad\left|\mathrm{F}_{2}^{\prime}(\mathrm{t})\right| \leq \frac{1+10 \varepsilon}{2 \mathrm{C}_{W}} \quad \text { and } \quad\left|\mathrm{F}_{2}^{\prime \prime}(\mathrm{t})\right|<5 / \varepsilon^{4} .
$$

(iii) Suppose $t \in\left[p_{i-1}, p_{i}\right]$ with $p_{i}-p_{i-1}<2 \delta / \varepsilon^{2}$. Then $F_{2}^{\prime}(t)=0$.

Proof. Let

$$
A=\{0, \varepsilon\} \cup\left\{p_{i} ; i=2, \ldots, k, p_{i}-p_{i-1}<2 \delta / \varepsilon^{2}\right\} .
$$

Then $\#(A) \leq k$. Let $0=q_{1}<q_{2}<\ldots<q_{\ell}=\varepsilon$ be an enumeration of the points of $A$, with $\ell \leq k$. We begin by constructing the function $F_{2}$ on the interval $[0, \varepsilon]$. To that end, we employ Lemma 2.4 for $t_{0}=0, t_{1}=\varepsilon$, for the function values $x_{0}=f_{2}(0), x_{1}=f_{2}(\varepsilon)$, for $\varepsilon^{3}$, and for the points $q_{1}, \ldots, q_{\ell}$. Note that

$$
\ell \varepsilon^{3} \leq k \varepsilon^{3} \leq \varepsilon^{2}<1 / 10 .
$$

Thus the appeal to Lemma 2.4 is legitimate. By the conclusion of that lemma, there exists a $\mathrm{C}^{2}$-function $\mathrm{F}_{2}:[0, \varepsilon] \rightarrow \mathbb{R}$ that satisfies:

(a) $F_{2}(\varepsilon i)=f_{2}(\varepsilon i)$ for $i=0,1$.

Moreover, $f_{2}(0) \leq F_{2}(t) \leq f_{2}(\varepsilon)$ for $t \in[0, \varepsilon]$.

(b) For any $i=1, . ., \ell$,

$$
F_{2}^{\prime}(t)=0 \quad \text { for } t \in\left[q_{i}-\varepsilon^{4}, q_{i}+\varepsilon^{4}\right] \cap[0, \varepsilon] .
$$

(c) For any $t \in[0, \varepsilon]$,

$$
\left|F_{2}^{\prime}(t)\right| \leq\left(1+10 \ell \varepsilon^{3}\right) \cdot\left(f_{2}(\varepsilon)-f_{2}(0)\right) / \varepsilon \leq \frac{1+10 \varepsilon^{2}}{2 C_{W}} .
$$

(d) For any $t \in[0, \varepsilon]$,

$$
\left|F_{2}^{\prime \prime}(t)\right| \leq 5 \varepsilon^{-3}\left(f_{2}(\varepsilon)-f_{2}(0)\right) / \varepsilon^{2} \leq 5 \varepsilon^{-4} .
$$

It remains to construct the function $F_{2}$ in the intervals $\left[-\varepsilon^{2}, 0\right)$ and $\left(\varepsilon, \varepsilon+\varepsilon^{2}\right]$.

We set $F_{2}$ to be constant in these two intervals. That is,

$$
\begin{aligned}
& F_{2}(t)=0 \text { for }-\varepsilon^{2} \leq t<0, \quad \text { and } \\
& F_{2}(t)=f_{2}(\varepsilon) \text { for } \varepsilon<t \leq \varepsilon+\varepsilon^{2} .
\end{aligned}
$$


This completes the definition of the function $F_{2}:\left[-\varepsilon^{2}, \varepsilon+\varepsilon^{2}\right] \rightarrow \mathbb{R}$. The function $F_{2}$ is $C^{2}$-smooth in $[0, \varepsilon]$. Moreover, $F_{2}^{\prime}=0$ on $\left[0, \varepsilon^{4}\right] \cup\left[\varepsilon-\varepsilon^{4}, \varepsilon\right]$. Since $F_{2}$ is constant on $\left[-\varepsilon^{2}, 0\right)$ and $\left(\varepsilon, \varepsilon+\varepsilon^{2}\right]$, then $F_{2}$ is a $C^{2}$-function on the entire interval $\left[-\varepsilon^{2}, \varepsilon+\varepsilon^{2}\right]$.

The function $F_{2}$ satisfies properties (i) and (ii) from the conclusion of the present lemma, because of $(\mathrm{a}),(\mathrm{c})$ and $(\mathrm{d})$. Note that $\mathrm{F}_{2}^{\prime}$ vanishes on an $\varepsilon^{4}$-neighborhood of any $q_{i}$, according to (b). Clearly, $\varepsilon^{4}>2 \delta / \varepsilon^{2}$. Therefore, $\mathrm{F}_{2}^{\prime}(\mathrm{t})=0$ if $\mathrm{t} \in\left[\mathrm{p}_{i-1}, \mathrm{p}_{i}\right]$ with $\mathrm{p}_{\mathrm{i}}-\mathrm{p}_{\mathrm{i}-1}<2 \delta / \varepsilon^{2}$. Hence property (iii) holds true. The proof is complete.

Proof of Lemma 5.1. Define $\mathrm{U}=\left(-\varepsilon^{2}, \varepsilon+\varepsilon^{2}\right) \times\left(-\varepsilon^{5}, \varepsilon^{5}\right)$ and $\mathrm{V}=$ $\left(-\varepsilon^{3}, \varepsilon+\varepsilon^{3}\right) \times\left(-\varepsilon^{6}, \varepsilon^{6}\right)$. Then $\mathrm{V} \subset \mathrm{U} \subset \mathbb{R}^{2}$. We define a $\mathrm{C}^{2}$-function $\tilde{\mathrm{F}}_{2}: \mathrm{U} \rightarrow \mathbb{R}$ by setting

$$
\tilde{F}_{2}(x, y)=y F_{2}(x) \quad \text { for }(x, y) \in U,
$$

where $F_{2}$ is the function constructed in Lemma 5.3. From Lemma 5.3 we know that for any $z \in \mathrm{U}$,

$$
\begin{aligned}
\left|\tilde{\mathrm{F}}_{2}(z)\right|,\left|\partial^{x} \tilde{\mathrm{F}}_{2}(z)\right|,\left|\partial^{y y} \tilde{\mathrm{F}}_{2}(z)\right| & \leq \varepsilon^{5} \\
\left|\partial^{y} \tilde{\mathrm{F}}_{2}(z)\right|,\left|\partial^{x x} \tilde{\mathrm{F}}_{2}(z)\right| & \leq 5 \varepsilon \\
\left|\partial^{x y} \tilde{\mathrm{F}}_{2}(z)\right| & \leq \frac{1+10 \varepsilon}{2 \mathrm{C}_{W}} .
\end{aligned}
$$

Consequently, our function $\tilde{\mathrm{F}}_{2}: \mathrm{U} \rightarrow \mathbb{R}$ satisfies $\left\|\tilde{\mathrm{F}}_{2}\right\|_{\mathrm{C}^{2}(\mathrm{U})} \leq \frac{1}{\sqrt{2} \mathrm{C}_{W}}+\mathrm{C} \varepsilon$, by the definition (1.3) of the $\mathrm{C}^{2}$ norm. We will use Whitney's theorem to extend $\tilde{F}_{2}$ to the entire $\mathbb{R}^{2}$. According to Lemma 2.1, there exists a $C^{2}$ function $\overline{\mathrm{F}}_{2}: \mathbb{R}^{2} \rightarrow \mathbb{R}$ such that

$$
\left\|\overline{\mathrm{F}}_{2}\right\|_{\mathrm{C}^{2}} \leq \mathrm{C}_{W}\left\|\tilde{\mathrm{F}}_{2}\right\|_{\mathrm{C}^{2}(\mathrm{U})} \leq \frac{1}{\sqrt{2}}+\mathrm{C}^{\prime} \varepsilon, \quad \text { and }\left.\quad \overline{\mathrm{F}}_{2}\right|_{\mathrm{V}}=\left.\tilde{\mathrm{F}}_{2}\right|_{\mathrm{v}}
$$

Let $\mathrm{I}: \mathbb{R} \rightarrow[0,1]$ be a $\mathrm{C}^{2}$-smooth cutoff function with the following properties:

1. $I(t)=1$ for $|t| \leq \frac{1}{2} \sqrt{\delta} / \varepsilon^{2}$.

2. $I(t)=0$ for $|t|>\sqrt{\delta} / \varepsilon^{2}$.

3. $\left|\mathrm{I}^{\prime}(\mathrm{t})\right|<\mathrm{C} \varepsilon^{2} / \sqrt{\delta},\left|\mathrm{I}^{\prime \prime}(\mathrm{t})\right|<\mathrm{C} \varepsilon^{4} / \delta$ for all $\mathrm{t} \in \mathbb{R}$.

There clearly exists such a function I. Next we define

$$
\overline{\mathrm{F}}_{1}(x, y)=\mathrm{F}_{1}(x) \mathrm{I}(\mathrm{y}) \quad \text { for }(x, y) \in \mathbb{R}^{2},
$$

where $F_{1}$ is the function from Lemma 5.2. 
Finally, we set for $(x, y) \in \mathbb{R}^{2}$,

$$
F(x, y)=\bar{F}_{1}(x, y)+\bar{F}_{2}(x, y) .
$$

Let us verify that $F$ satisfies the three assertions of the lemma. Recall the definition of the set $T$ from the beginning of this section. Suppose that $z=(x, 0) \in \mathrm{T}$. Then $(x, 0) \in \mathrm{V}$ and hence

$$
\mathrm{F}(x, 0)=\overline{\mathrm{F}}_{1}(x, 0)+\overline{\mathrm{F}}_{2}(x, 0)=\mathrm{F}_{1}(x) \mathrm{I}(0)+\tilde{F}_{2}(x, 0)=\mathrm{F}_{1}(x)=\mathrm{f}_{1}(x)=\mathrm{f}(x, 0)
$$

by Lemma 5.2. This proves the first assertion of the lemma.

Next, suppose that $z=(x, \delta) \in \ell_{2}$. Then $x=0$ or $x=\varepsilon$. Also, $\delta<\varepsilon^{6}$ and $0 \leq x \leq \varepsilon$, hence $(x, \delta) \in \mathrm{V}$. Now,

$\mathrm{F}(x, \delta)=\overline{\mathrm{F}}_{1}(x, \delta)+\overline{\mathrm{F}}_{2}(x, \delta)=\mathrm{F}_{1}(x) \mathrm{I}(\delta)+\tilde{\mathrm{F}}_{2}(x, \delta)=\mathrm{f}_{1}(x)+\delta \mathrm{f}_{2}(x)=\mathrm{f}(\mathrm{x}, \delta)$

according to Lemma 5.2 and Lemma 5.3 , where $\mathrm{I}(\delta)=1$ as $\delta \leq \frac{1}{2} \sqrt{\delta} / \varepsilon^{2}$. This proves the second assertion of the lemma.

It remains to show that

$$
\|\mathrm{F}\|_{C^{2}} \leq 1
$$

That is, we need to prove that

$$
\begin{aligned}
\left|\mathrm{F}\left(z_{0}\right)\right|^{2}+\left|\partial^{x} \mathrm{~F}\left(z_{0}\right)\right|^{2} & +\left|\partial^{y} \mathrm{~F}\left(z_{0}\right)\right|^{2}+\left|\partial^{x x} \mathrm{~F}\left(z_{0}\right)\right|^{2} \\
& +2\left|\partial^{x y} \mathrm{~F}\left(z_{0}\right)\right|^{2}+\left|\partial^{y y} \mathrm{~F}\left(z_{0}\right)\right|^{2} \leq 1
\end{aligned}
$$

for all $z_{0}=\left(x_{0}, y_{0}\right) \in \mathbb{R}^{n}$. To that end, we will use the estimates from Lemma 5.2 and from Lemma 5.3. Fix a point $z_{0}=\left(x_{0}, y_{0}\right) \in \mathbb{R}^{2}$.

Case 1: Suppose that $z_{0} \in V$. Then for $z=(x, y)$ in a neighborhood of $z_{0}$,

$$
\mathrm{F}(z)=\overline{\mathrm{F}}_{1}(z)+\overline{\mathrm{F}}_{2}(z)=\mathrm{I}(\mathrm{y}) \mathrm{F}_{1}(\mathrm{x})+\mathrm{y} \mathrm{F}_{2}(\mathrm{x}) .
$$

We know that $\left|\mathrm{F}\left(z_{0}\right)\right|<\mathrm{C} \varepsilon$, as $\left|\mathrm{F}_{1}\left(\mathrm{x}_{0}\right)\right|,\left|\mathrm{F}_{2}\left(\mathrm{x}_{0}\right)\right|$, $\left|\mathrm{y}_{0}\right|<\mathrm{C}^{\prime} \varepsilon$ while $\left|\mathrm{I}\left(\mathrm{y}_{0}\right)\right| \leq 1$. Regarding first derivatives, note that

$$
\partial^{x} \mathrm{~F}\left(z_{0}\right)=\mathrm{I}\left(\mathrm{y}_{0}\right) \mathrm{F}_{1}^{\prime}\left(x_{0}\right)+\mathrm{y}_{0} \mathrm{~F}_{2}^{\prime}\left(\mathrm{x}_{0}\right), \quad \partial^{y} \mathrm{~F}\left(z_{0}\right)=\mathrm{I}^{\prime}\left(\mathrm{y}_{0}\right) \mathrm{F}_{1}\left(\mathrm{x}_{0}\right)+\mathrm{F}_{2}\left(\mathrm{x}_{0}\right) .
$$

We have $\left|\mathrm{F}_{1}\left(\mathrm{x}_{0}\right)\right|,\left|\mathrm{F}_{1}^{\prime}\left(\mathrm{x}_{0}\right)\right|<5 \delta / \varepsilon^{2}$ while $\left|\mathrm{y}_{0}\right|<\varepsilon^{6},\left|\mathrm{I}\left(\mathrm{y}_{0}\right)\right| \leq 1,\left|\mathrm{I}^{\prime}\left(\mathrm{y}_{0}\right)\right|<$ $C \varepsilon^{2} \delta^{-1 / 2}$ and $\left|F_{2}\left(x_{0}\right)\right|<5 \varepsilon,\left|F_{2}^{\prime}\left(x_{0}\right)\right|<1$. Therefore, the contribution of the first derivatives to the sum in (5.9) is bounded by $\mathrm{C} \varepsilon^{2}$.

We move to the second derivatives. Firstly,

$$
\partial^{y y} \mathrm{~F}\left(z_{0}\right)=I^{\prime \prime}\left(y_{0}\right) F_{1}\left(x_{0}\right) .
$$

Since $\left|\mathrm{I}^{\prime \prime}\left(\mathrm{y}_{0}\right)\right|<\mathrm{C} \varepsilon^{4} / \delta$ with $\left|\mathrm{F}_{1}\left(\mathrm{x}_{0}\right)\right|<\mathrm{C} \delta / \varepsilon^{2}$, then $\left|\partial^{y y} \mathrm{~F}\left(z_{0}\right)\right|<\mathrm{C}^{\prime} \varepsilon^{2}$. 
Next,

$$
\left|\partial^{x y} F\left(z_{0}\right)\right|=\left|I^{\prime}\left(y_{0}\right) F_{1}^{\prime}\left(x_{0}\right)+F_{2}^{\prime}\left(x_{0}\right)\right| \leq C \varepsilon+\left|F_{2}^{\prime}\left(x_{0}\right)\right|
$$

since $\left|\mathrm{I}^{\prime}\left(\mathrm{y}_{0}\right)\right|<\mathrm{C} \varepsilon^{2} / \sqrt{\delta}$ with $\left|\mathrm{F}_{1}^{\prime}\left(\mathrm{x}_{0}\right)\right|<\mathrm{C} \delta / \varepsilon^{2}$.

In addition,

$$
\left|\partial^{x x} \mathrm{~F}\left(z_{0}\right)\right|=\left|\mathrm{I}\left(\mathrm{y}_{0}\right) \mathrm{F}_{1}^{\prime \prime}\left(\mathrm{x}_{0}\right)+\mathrm{y}_{0} \mathrm{~F}_{2}^{\prime \prime}\left(\mathrm{x}_{0}\right)\right| \leq \mathrm{C} \varepsilon+\left|\mathrm{F}_{1}^{\prime \prime}\left(\mathrm{x}_{0}\right)\right|
$$

because $\left|y_{0}\right|<\varepsilon^{6},\left|F_{2}^{\prime \prime}\left(x_{0}\right)\right|<5 \varepsilon^{-4}$ and $\left|\mathrm{I}\left(y_{0}\right)\right| \leq 1$. By combining the estimates obtained so far, we conclude that the sum in (5.9) is bounded by

$$
\left(\mathrm{C} \varepsilon+\left|\mathrm{F}_{1}^{\prime \prime}\left(\mathrm{x}_{0}\right)\right|\right)^{2}+2\left(\mathrm{C} \varepsilon+\left|\mathrm{F}_{2}^{\prime}\left(\mathrm{x}_{0}\right)\right|\right)^{2} .
$$

Therefore, to establish (5.9) in the case $z_{0} \in \mathrm{V}$, it is sufficient to show that

$$
\left|F_{1}^{\prime \prime}\left(x_{0}\right)\right|^{2}+2\left|F_{2}^{\prime}\left(x_{0}\right)\right|^{2} \leq\left(C_{W}^{\prime}+C \varepsilon\right)^{2} .
$$

Indeed, $C_{W}^{\prime}=1-10^{-6} / C_{W}^{4}$ and $C_{W}^{\prime}+C \varepsilon \leq 1$ under the legitimate assumption that $\mathrm{k}^{\#}$ exceeds a certain universal constant. Thus, we focus on proving (5.10).

Suppose first that $x_{0} \in\left[p_{i-1}, p_{i}\right]$ with $p_{i}-p_{i-1}<2 \delta / \varepsilon^{2}$. In this case

$$
\mathrm{F}_{2}^{\prime}\left(\mathrm{x}_{0}\right)=0, \quad \text { and } \quad\left|\mathrm{F}_{1}^{\prime \prime}\left(\mathrm{x}_{0}\right)\right| \leq \mathrm{C}_{W}^{\prime}+\varepsilon
$$

so (5.10) holds.

It remains to deal with the case where one of the following three possibilities holds true: Either $x_{0}>\varepsilon$, or else $x_{0}<0$ or else $x_{0} \in\left[p_{i-1}, p_{i}\right]$ with $p_{i}-p_{i-1} \geq 2 \delta / \varepsilon^{2}$. In all three possibilities, we have that $\left|F_{1}^{\prime \prime}\left(x_{0}\right)\right|<\varepsilon$. Since always

$$
\left|\mathrm{F}_{2}^{\prime}\left(\mathrm{x}_{0}\right)\right|<\frac{1}{2 \mathrm{C}_{W}}(1+10 \varepsilon)<\frac{3}{5}
$$

then (5.10) holds also in this case (recall that $\left.C_{W}^{\prime}>9 / 10\right)$. Therefore (5.10) holds in all cases. This completes the proof of (5.9) in the case where $z_{0} \in \mathrm{V}$.

Case 2: Suppose $z_{0}=\left(x_{0}, y_{0}\right)$ is such that $\left|y_{0}\right| \geq \varepsilon^{6}$. Then $I(y)$ vanishes in a neighborhood of $y_{0}$. Consequently, $F=\bar{F}_{2}$ in a neighborhood of $z$, and (5.9) follows from the bound

$$
\left\|\overline{\mathrm{F}}_{2}\right\|_{\mathrm{C}^{2}} \leq \frac{1}{\sqrt{2}}+\mathrm{C} \varepsilon
$$

in (5.8). 
Case 3: Suppose $z=\left(x_{0}, y_{0}\right)$ is such that $x_{0}<0$ or $x_{0}>\varepsilon$. In this case, Lemma 5.2 states that $\left|F_{1}^{\prime \prime}\left(x_{0}\right)\right| \leq \varepsilon$. Therefore, $\left|\partial^{x x} \bar{F}_{1}\left(z_{0}\right)\right| \leq \varepsilon$. The other derivatives of $\bar{F}_{1}$ are bounded as well; reasoning as in Case 1 we see that

$$
\left|\overline{\mathrm{F}}_{1}\left(z_{0}\right)\right|,\left|\partial^{x} \overline{\mathrm{F}}_{1}\left(z_{0}\right)\right|,\left|\partial^{y} \overline{\mathrm{F}}_{1}\left(z_{0}\right)\right|\left|\partial^{y y} \overline{\mathrm{F}}_{1}\left(z_{0}\right)\right|\left|\partial^{x y} \overline{\mathrm{F}}_{1}\left(z_{0}\right)\right|<C \varepsilon
$$

Recall that $F=\bar{F}_{1}+\bar{F}_{2}$, and that $\left\|\bar{F}_{2}\right\|_{C^{2}} \leq \frac{1}{\sqrt{2}}+C \varepsilon$, by (5.8). We therefore conclude from our bounds on the derivatives of $\bar{F}_{1}$ that the sum in (5.9) is controlled by

$$
\left(\left\|\overline{\mathrm{F}}_{2}\right\|_{\mathrm{C}^{2}}+\mathrm{C} \varepsilon\right)^{2} \leq \frac{1}{2}+\mathrm{C}^{\prime} \varepsilon
$$

Therefore (5.9) holds.

Note that any $z_{0} \in \mathbb{R}^{2}$ falls into Case 1 , Case 2 or Case 3. Hence (5.9) is established in all cases, and the lemma is proven.

Proof of Proposition 1.3. We may assume that $k^{\#}$ is a sufficiently large integer. Given $k^{\#}$, we have constructed in Section 3 a certain finite set $E \subset \mathbb{R}^{2}$ and a function $f: E \rightarrow \mathbb{R}$. In section 4 , the first assertion of the present proposition was verified, with the universal constant $c_{0}=10^{-4} / C_{W}^{3}>0$. In the present section, we verified the second assertion; see Lemma 5.1 and the sentence following it. This completes the proof.

\section{Comments}

Proposition 1.3 was stated and proved for a particular choice of the $\mathrm{C}^{2}$ norm. Namely,

$$
\begin{gathered}
\|f\|_{C^{2}}^{2}=\sup _{z \in \mathbb{R}^{2}}\left\{|f(z)|^{2}+\left|\partial^{x} f(z)\right|^{2}+\left|\partial^{y} f(z)\right|^{2}+\left|\partial^{x x} f(z)\right|^{2}\right. \\
\left.+2\left|\partial^{x y} f(z)\right|^{2}+\left|\partial^{y y} f(z)\right|^{2}\right\} .
\end{gathered}
$$

Nevertheless, the counter-example we presented is quite general and robust, with respect to modifications of the definition of the $\mathrm{C}^{2}$ norm.

The zero-derivative and the first-derivative parts of the $C^{2}$ norm did not have much influence here: Throughout all of the analysis, their contribution to the $\mathrm{C}^{2}$ norm was bounded by $\mathrm{C} \varepsilon$. Thus, any reasonable modification of the zero-derivative and the first-derivative parts in the definition of the $C^{2}$ norm should not affect the proof.

The main term in the $\mathrm{C}^{2}$ norm is the second derivatives. Essentially, we used only the following property of the $C^{2}$ norm: For $z \in \mathbb{R}^{2}$ close to the 
origin, with respect to some orthogonal coordinates in $\mathbb{R}^{2}$,

$$
\begin{gathered}
\left\|\left(\begin{array}{cc}
\approx 1 & \approx 0 \\
\approx 0 & \approx 0
\end{array}\right)\right\| \approx 1,\left\|\left(\begin{array}{ll}
\approx 0 & \approx c \\
\approx c & \approx 0
\end{array}\right)\right\|<1-c_{0}, \\
\text { but }\left\|\left(\begin{array}{rr}
\approx 1 & \approx c \\
\approx c & \approx 0
\end{array}\right)\right\|>1+c_{0}
\end{gathered}
$$

for some small universal constants $c, c_{0}>0$ where $\|A\|$ stands for the contribution of the hessian matrix $A=\operatorname{Hess}(F)(z)$ to the $C^{2}$ norm.

There are plenty of $C^{2}$ norms that satisfy (6.1) with respect to an appropriate choice of an orthogonal basis in $\mathbb{R}^{2}$; Thus, our counter-example should work for many reasonable $\mathrm{C}^{2}$ norms, including, for instance, the definition (1.1).

Our finite set $E$ was defined as the union of $\ell_{1}$ and $\ell_{2}$. The set $\ell_{1}$ consists of many equidistant points on a line, while $\ell_{2}$ has only two points in it. What happens if we remove these two points from the set $E$ ? We conclude this article by remarking that there is no counter-example for the regular, one-dimensional set $\ell_{1}$, if we confine ourselves to the specific definition (1.3) of the $\mathrm{C}^{2}$ norm. The details are omitted.

\section{References}

[1] Bierstone, E., Milman, P. and Paweucki, W.: Differentiable functions defined on closed sets. A problem of Whitney. Invent. Math. 151 (2003), no. 2, 329-352.

[2] Bierstone, E., Milman, P. And PawŁucki, W.: Higher-order tangents and Fefferman's paper on Whitney's extension problem. Ann. of Math. (2) 164 (2006), no. 1, 361-370.

[3] Brudnyi, A. And Brudnyi, Y.: Metric spaces with linear extensions preserving Lipschitz condition. Amer. J. Math. 129 (2007), no. 1, 217-314.

[4] Brudnyi, Y.: On an extension theorem. Funk. Anal. i Prilzhen. 4 (1970), 97-98; English transl. in Func. Anal. Appl. 4 (1970), 252-253.

[5] Brudnyi, Y. and Shvartsman, P.: The traces of differentiable functions to closed subsets of $\mathbb{R}^{n}$. In Function Spaces (Poznán, 1989), 206-210. Teubner-Texte Math. 120. Teubner, Stuttgart, 1991.

[6] Brudnyi, Y. And Shvartsman, P.: A linear extension operator for a space of smooth functions defined on closed subsets of $\mathrm{R}^{\mathrm{n}}$. Dokl. Akad. Nauk SSSR 280 (1985), 268-270. English transl. in Soviet Math. Dokl. 31 (1985), no. 1, 48-51.

[7] Brudnyi, Y. and Shvartsman, P.: Generalizations of Whitney's extension theorem. Int. Math. Research Notices 3 (1994), 129-139. 
[8] Brudnyi, Y. and Shvartsman, P.: The Whitney problem of existence of a linear extension operator. J. Geom. Anal. 7 (1997), no. 4, 515-574.

[9] Brudnyi, Y. and Shvartsman, P.: Whitney's extension problem for multivariate $C^{1, w}$ functions. Trans. Amer. Math. Soc. 353 (2001), no. 6, $2487-2512$.

[10] Fefferman, C.: Interpolation and extrapolation of smooth functions by linear operators. Rev. Mat. Iberoamericana 21 (2005), no. 1, 313-348.

[11] Fefferman, C.: A sharp form of Whitney's extension theorem. Ann. of Math. (2) 161 (2005), 509-577.

[12] Fefferman, C.: Whitney's extension problem for $\mathrm{C}^{\mathrm{m}}$. Ann. of Math. (2) 164 (2006), no. 1, 313-359.

[13] Fefferman, C.: Whitney's extension problem in certain function spaces. (preprint).

[14] Fefferman, C.: A generalized sharp Whitney theorem for jets. Rev. Mat. Iberoamericana 21 (2005), no. 2, 577-688.

[15] Fefferman, C.: Extension of $\mathrm{C}^{\mathrm{m}, \omega}$ smooth functions by linear operators. Rev. Mat. Iberoamericana 25 (2009), no. 1, 1-48.

[16] Fefferman, C.: $\mathrm{C}^{\mathrm{m}}$ extension by linear operators Ann. of Math. (2) 166 (2007), no. 3, 779-835.

[17] Fefferman, C.: Fitting a $\mathrm{C}^{\mathrm{m}}$-smooth function to data III, to appear in Ann. of Math. (2).

[18] Fefferman, C.: The $\mathrm{C}^{\mathrm{m}}$ norm of a function with prescribed jets $\mathrm{I}$, preprint.

[19] Fefferman, C.: The $\mathrm{C}^{\mathrm{m}}$ norm of a function with prescribed jets II. Rev. Mat. Iberoamericana 25 (2009), no. 1, 275-421.

[20] Fefferman, C. and Klartag, B.: Fitting a $\mathrm{C}^{\mathrm{m}}$-smooth function to data I. Annals of Math. (2) 169 (2009), no. 1, 315-346.

[21] Fefferman, C. And Klartag, B.: Fitting a $\mathrm{C}^{\mathrm{m}}$-smooth function to data II. Rev. Mat. Iberoamericana 25 (2009), no. 1, 49-273.

[22] Glaeser, G.: Étude de quelques algèbres tayloriennes. J. Analyse Math. 6 (1958), 1-124.

[23] Shvartsman, P.: Lipschitz selections of multivalued mappings and traces of the Zygmund class of functions to an arbitrary compact. Dokl. Acad. Nauk SSSR 276 (1984), 559-562; English transl. in Soviet Math. Dokl. 29 (1984), 565-568.

[24] Shvartsman, P.: On traces of functions of Zygmund classes. Sibirskyi Mathem. J. 28 N5 (1987), 203-215; English transl. in Siberian Math. J. 28 (1987), 853-863.

[25] Shvartsman, P.: Lipschitz selections of set-valued functions and Helly's theorem. J. Geom. Anal. 12 (2002), no. 2, 289-324.

[26] Stein, E. M.: Singular Integrals and Differentiability Properties of Functions. Princeton Univ. Press, 1970. 
[27] Whitney, H.: Analytic extensions of differentiable functions defined in closed sets. Trans. Amer. Math. Soc. 36 (1934), 63-89.

[28] Whitney, H.: Differentiable functions defined in closed sets I. Trans. Amer. Math. Soc. 36 (1934), 369-389.

[29] Whitney, H.: Functions differentiable on the boundaries of regions. Ann. of Math. 35 (1934), 482-485.

[30] Zobin, N.: Whitney's problem on extendability of functions and an intrinsic metric. Adv. Math. 133 (1998), no. 1, 96-132.

[31] Zobin, N.: Extension of smooth functions from finitely connected planar domains. J. Geom. Anal. 9 (1999), no. 3, 489-509.

Recibido: 10 de abril del 2007

Charles Fefferman

Department of Mathematics Princeton University, Fine Hall

Washington Road

Princeton, New Jersey 08544, USA cf@math.princeton.edu

Bo'az Klartag

School of Mathematics

Institute for Advanced Study

Einstein Drive

Princeton, New Jersey 08540, USA

klartag@ias.edu

First author is partially supported by NSF grant \#DMS-0245242. Second author is supported by the Clay Mathematics Institute and by NSF grant \#DMS-0456590. 